\title{
Über den Zusammenhang von Wissen und Anwendung kompetenzorientierter Leistungsfeststellungs- und Leistungsbeurteilungsformate
}

Eine Erhebung an niederösterreichischen Berufsschulen

\author{
Elena Lehmann ${ }^{1}$, Franz Erhard ${ }^{2}$
}

https://doi.org/10.53349/resource.2021.i16.a995

\section{Zusammenfassung}

Dieser Artikel stellt die Ergebnisse einer empirisch-quantitativen Untersuchung zu kompetenzorientierter Leistungsfeststellung und Leistungsbeurteilung an niederösterreichischen Berufsschulen vor. Dabei baut der Fragebogen, der dieser Datensammlung zugrunde liegt, im Sinne einer sequentiellen Nachschau (Teddlie und Tashakkori, 2006, S. 153) auf den Ergebnissen einer vorangegangenen qualitativen Erhebung auf (Lehmann \& Erhard, 2020).

Im Zuge der zuvor durchgeführten qualitativen Untersuchung verdichtete sich die Annahme, dass die aktuell angewendeten Prüfungsformate und Beurteilungsinstrumente die Implementierung kompetenzorientierter Unterrichtsentwicklung, wie sie seit 2012 auch an niederösterreichischen Berufsschulen eingeführt wurde, unzureichend widerspiegeln (ebd., S.10). Nach Auswertung der quantitativen Fragebogenerhebung konnte eine teils eingeschränkte Umsetzung von vorhandenem Wissen um kompetenzorientierte Leistungsfeststellung und Leistungsbeurteilung bestätigt werden. Somit kann angenommen werden, dass der tatsächliche Handlungsspielraum, wie ihn die Leistungsbeurteilungsverordnung (LBVO) in Bezug auf Kompetenzorientierung vorsieht, nicht in seinem vollen Umfang ausgeschöpft wird.

\begin{tabular}{ll}
\hline Schlüsselwörter: & Keywords: \\
Berufsschule & Vocational School \\
Kompetenzorientierung & Competence Orientation \\
Leistungsfeststellung & Student performance assessment \\
Leistungsbeurteilung & Student performance evaluation \\
Leistungsbeurteilungsverordnung (LBVO) & Performance Appraisal Ordinance
\end{tabular}

\section{Einleitung}

In dem vorliegenden Artikel wird der zweite Teil einer Untersuchung zu kompetenzorientierter Leistungsfeststellung und Leistungsbeurteilung an niederösterreichischen Berufsschulen vorgestellt. Dieser empirisch-quantitative Teil erläutert die Auswertung einer umfassenden Befragung von Lehrkräften an niederösterreichischen Berufsschulen. Im Anschluss daran wurden die dabei gewonnenen Daten mit den

1 Landesberufsschule Baden, Josef-Kollmann Straße 1, 2500 Baden und Pädagogische Hochschule Niederösterreich, Mühlgasse 67, 2500 Baden.

E-Mail:E.Lehmann@ph-noe.ac.at

${ }^{2}$ Pädagogische Hochschule Niederösterreich, Mühlgasse 67, 2500 Baden.

E-Mail: Franz.Erhard@ph-noe.ac.at 
Ergebnissen aus sechs leitfadengestützten Einzelinterviews aus der vorangegangenen qualitativen Erhebung verglichen. Mit der Gegenüberstellung der Auswertungsergebnisse beider Datensammlungen konnte der zentralen Frage nachgegangen werden, welches Wissen in Bezug auf kompetenzorientierte Leistungsfeststellung und Leistungsbeurteilung an niederösterreichischen Berufsschulen vorhanden ist und ob und wie das vorhandene Wissen zur Anwendung gebracht wird.

Wie bereits in dem Artikel Über den Umgang mit schulischer Leistung unter kompetenzorientierten Gesichtspunkten von Lehmann \& Erhard (2020) ausgeführt, wurden seit 2012 Unterrichtsmethoden und Lehrmaterialien an niederösterreichischen Berufsschulen gemäß der vom Gesetzgeber verordneten kompetenzorientierten Rahmenlehrpläne so aufbereitet, dass die Lehrinhalte heute ein hohes Potential an Praxisbezogenheit und Differenzierung aufweisen (S.1 f.).

Dabei lenken die Autorin und der Autor einen besonderen Blick auf die Arbeit der Lehrkräfte mit Schüler*innen, deren Lernausgangslagen im Vergleich unterschiedliche Kompetenzniveaus aufweisen. Dieser Umstand ist vor allem in der Berufsschule einer überdurchschnittlich hohen Heterogenität hinsichtlich des Alters, der Vorbildung, der kulturellen Hintergründe sowie der (bildungs-)sprachlichen Ressourcen geschuldet. Diese Vielfalt lässt sich auf die Tatsache zurückführen, dass im österreichweiten Durchschnitt $40 \%$ aller Jugendlichen mit unterschiedlichen formalen und informellen Bildungsverläufen die Berufsschule als Teil einer dualen Lehrausbildung besuchen (Dornmayer \& Nowak, 2019, S.23).

Mit der österreichweiten Implementierung kompetenzorientierter Unterrichtskonzepte im vergangenen Jahrzehnt wurde diese Komplexität einer umfassenden Anerkennung zugeführt.

Jedoch scheint das Prinzip der Kompetenzorientierung auf den ersten Blick in der Leistungsbeurteilungsverordnung (LBVO) 1974 i.d.g.F. explizit wenig Berücksichtigung zu finden, wenn, wie in dieser, eine sachbezogene, lehrzielorientierte Bezugsnorm als Leistungsstandard vorgegeben wird (LBVO § 11 Abs. 1, zweiter Satz i.d.g.F).

Diese Annahme bildete den Ausgangspunkt für die hier vorgestellte Studie, die der zentralen Frage nachging, ob und in welcher Form Leistungsfeststellung und Leistungsbeurteilung an niederösterreichischen Berufsschulen kompetenzorientierten Maßgaben folgt. Darüber hinaus wurde auch möglichen Ursachen für die durch die Datenerhebung sichtbar gewordene eingeschränkte Umsetzung kompetenzorientierter Leistungsfeststellung und Leistungsbeurteilung nachgegangen. Nach erfolgter Auswertung der empirisch-qualitativen Einzelinterviews wurde ein Fragebogen entwickelt, dessen Ergebnisse der hier dargestellten quantitativen Erhebung zugrunde liegen (siehe Abschnitt 3, Design und Ablauf).

Ziel dieser zweiten Untersuchung war es, die in der qualitativen Analyse gewonnenen Aussagen zu bestätigen oder zu widerlegen.

\section{Theoretische Anbindung}

Die folgenden Ausführungen basieren auf den Inhalten von Abschnitt 2 des zitierten Artikels von Lehmann und Erhard (Lehmann \& Erhard, 2020) und werden daher in Folge zusammenfassend dargestellt.

\subsection{Kompetenzorientierter Unterricht in der Berufsschule}

In Österreich ist die Berufsschule Teil eines dualen Ausbildungssystems, das zur Aufgabe hat, vornehmlich junge Menschen nach Beendigung der allgemeinbildenden Pflichtschule in einer berufspraktischen Ausbildung zu fördern und die betriebliche Ausbildung zu ergänzen sowie die Allgemeinbildung zu erweitern. Das gemeinsame Ziel der betrieblichen und schulischen Ausbildung ist die Ausbildung zu Facharbeiter*innen sowie die Heranführung zur Lehrabschlussprüfung, die in der Regel nach 2 bis 4 Jahren Lehrzeit abzulegen ist. Im Schuljahr 2019/20 wurden insgesamt 143 Berufsschulen im Bundesgebiet gezählt (Mohr, 2021).

Der dem Schultyp der Berufsschule eigene Erfahrungsvorsprung in Bezug auf die praktische Umsetzung theoretischer Inhalte mit möglichst hoher Nähe zur Arbeitswelt entspricht den Forderungen, die im Grundlagenpapier Kompetenzorientiertes Unterrichten an berufsbildenden Schulen des Bildungsministeriums aus 
dem Jahre 2012 an dieses Unterrichtsprinzip gestellt werden: „Kompetenzorientierter Unterricht zeichnet sich durch Handlungs- und Anwendungsorientiertheit sowie durch eine hohe aufgabenbezogene Aktivität auf Seiten der Lernenden aus" (Lehmann \& Erhard, S.12).

Zu diesem Zwecke orientieren sich die Fachkoordinator*innen sowie die Fachlehrer*innen bei der Erstellung der kompetenzorientierten Lehrpläne und Unterrichtsmaterialien gleichermaßen an den Notwendigkeiten gesetzlicher Vorgaben als auch an den Bedürfnissen der Wirtschaft, um die geforderte Nähe zur Berufswelt gewährleisten zu können.

Das übergeordnete Ziel ist es, mit den so erlernten Kompetenzen und Fähigkeiten den Forderungen nationaler und internationaler Standards zu entsprechen, die einen flexiblen Transfer von erworbenen Ausbildungspunkten im Rahmen des European Credit System for Vocational Education and Training (ECVET) erleichtern soll, um so größtmögliche Mobilität am europäischen und internationalen Arbeitsmarkt zu ermöglichen.

The purpose of this Recommendation is to create a European Credit System for Vocational Education and Training ('ECVET') intended to facilitate the transfer, recognition and accumulation of assessed learning outcomes of individuals who are aiming to achieve a qualification. This will improve the general understanding of citizens' learning outcomes and their transparency, transnational mobility and portability across and, where appropriate, within Member States in a borderless lifelong learning area, and will also improve the mobility and portability of qualifications at national level between various sectors of the economy and within the labour market; furthermore, it will contribute to the development and expansion of European cooperation in education and training. (Cedefop, 2009, C.155/11 in Lehmann \& Erhard, 2020)

Jedoch steht diese Empfehlung für länderübergreifende Leistungsstandards zum Zwecke der leichteren Vergleichbarkeit vordergründig im Widerspruch zu formativer, individualisierter Bewertung und Beurteilung von Leistung im Rahmen des kompetenzorientierten Unterrichts. Daher galt es, diesen Bereich wie folgt näher zu beleuchten.

\subsection{Kompetenzorientierte Leistungsfeststellung und Leistungsbeurteilung}

Grundlegend für die Leistungsbeurteilung und Leistungsfeststellung von kompetenzorientiert aufbereitetem Unterricht sind formative Instrumente der Lernentwicklung und Leistungsrückmeldung zur individuellen Förderung und Differenzierung. Im Gegensatz dazu steht die bildungsorientierte, summative Leistungsbeurteilung, die mit Notenziffern eine Zusammenfassung der Ergebnisse abgelegter Prüfungsformate in ihrer Gesamtheit, gemessen an der sachlichen Bezugsnorm des Rahmenlehrplans, darstellt. Dazu sagt der Leiter der Abteilung für Wirtschafts- und Berufspädagogik an der Johannes Kepler Universität Linz, Georg Neuweg:

Für die Ermittlung einer Gesamtnote, die den Notendefinitionen der LBVO gerecht wird, ist eine Übersicht über die erreichten Lehrziele und das Niveau, auf dem sie erreicht werden, erforderlich. Die in der Praxis verbreiteten Beurteilungssysteme wie etwas das schlichte Addieren von Punkten oder das Errechnen von Semester- oder Jahresnoten aus Einzelnoten leisten dies nicht (Neuweg, 2019, S.57).

Diese hier dargestellte Praxis fördert eine ausgrenzende Wirkung, da die individuelle Bezugsnorm, die Lernausgangslagen und individuellen Entwicklungsfortschritte Gefahr laufen, wenig Berücksichtigung zu finden. Gleichzeitig weist Neuweg mit diesem Zitat darauf hin, dass die LBVO durchaus das Prinzip der Individualisierung von Lernergebnissen und Kompetenzniveaus in die Feststellung und Beurteilung von Leistung miteinbeziehen kann. Da frühestens im Schuljahr 2021/22 mit der Veröffentlichung einer explizit kompetenzorientierten Leistungsbeurteilungsverordnung zu rechnen ist (Neuweg, 2019), lohnt es sich, die tatsächlichen Fördermöglichkeiten, die die aktuelle Fassung der Leistungsbeurteilungsverordnung LBVO (1974 i.d.g.F.) bietet, nochmals in Erinnerung zu rufen:

Ein wesentliches Merkmal kompetenzorientierter Leistungsfeststellung und Leistungsbeurteilung besteht darin, das Dilemma zwischen Fördern und Beurteilen wahrzunehmen und transparent mit den Schülerinnen und Schülern zu behandeln. Dies kann gelingen, wenn Beurteilungssituationen, die in leistungsheterogenen 
Klassen ein hohes Potential für Selektionserfahrungen bergen, eindeutig als solche gekennzeichnet werden und von den eigentlichen Lern- und Fördererfahrung organisatorisch getrennt werden (Neuweg, 2019, S.167 in Lehmann \& Erhard, 2020).

Dazu nennt Neuweg als wesentliches Instrument der kompetenzorientierten Leistungsfeststellung in Übereinstimmung mit den aktuellen gesetzlichen Vorgaben der LBVO (1974, i.d.g.F.) die Mitarbeitsfeststellung, da diese als alleinige Grundlage einer Semester- oder Jahresbeurteilung herangezogen werden kann, sofern der Unterrichtsgegenstand keine Schularbeit verlangt. Damit ist die Mitarbeitsfeststellung allen anderen Leistungsfeststellungsformaten gleichwertig (Neuweg, 2019, S.19f) und kann im Sinne der langfristigen Bewertung des Lernweges zur Benotung herangezogen werden.

Durch diese formative Art der Leistungsfeststellung kann die Notenfindung über einen längeren Zeitraum erfolgen und damit die individuelle Leistungsentwicklung der Schüler*innen in Bezug auf das Lehrziel festgehalten werden. Dabei ist vor allem ein Augenmerkt auf die Anwendbarkeit von theoretischem Wissen in der praktischen Arbeit zu legen. In der Berufsschule kann die Mitarbeitsfeststellung über die Produktherstellung im Werkstättenunterricht, über die Erstellung graphischer Arbeiten in Unterrichtsgegenständen wie Fachzeichnen, aber auch über die Anfertigung von Portfolios oder Projektberichten im Rahmen eines Projektpraktikums oder einer Übungsfirma genannt werden. (Lehmann \& Erhard, 2020, S.5)

In weiterer Folge bietet ein Kompetenzraster sowohl als Rückmeldeinstrument als auch als Grundlage zur Notenfindung die Möglichkeit der Einbeziehung von individuellen Lernausgangslagen. Zusätzlich kommt dem Kompetenzraster eine Förder- und Entwicklungsfunktion zu, indem die einzelnen Notenschritte des traditionellen Notenschlüssels in Kompetenzstufen ausgedrückt werden, was vom Gesetzgeber auch diesbezüglich empfohlen wird:

Die Förderfunktion (des Kompetenzrasters, Anm. der Autorin und des Autors) soll durch konkrete Vergleichsmaßstäbe, die bestmögliche Diagnostik als Grundlage für die Förderung einzelner Schüler*innen sicherstellen. Die damit einhergehende Entwicklungsfunktion schafft die Grundlage für einen kompetenzund schülerorientierten Unterricht (BMUKK, 2012, S.13, ebd.).

Mitarbeitsfeststellung und Kompetenzraster nehmen in Bezug auf den formativen Umgang mit Leistung eine herausragende Rolle ein. Daher dienen beide Instrumente in dieser Untersuchung als Indikatoren kompetenzorientierter Leistungsfeststellung und Leistungsbeurteilung, wenn in der hier nachfolgend dargestellten quantitativen Untersuchung ein besonderer Fokus auf den Nachweis von Wissen und Anwendung in Bezug auf Leistungsfeststellung und -beurteilung im kompetenzorientierten Kontext gelegt wird.

\section{Stand der Forschung}

Im Bereich der kompetenzorientierten Leistungsfeststellung und Leistungsbeurteilung liegen bislang keine vergleichbaren Untersuchungen innerhalb des Schultyps Berufsschule im deutschsprachigen Raum vor. Daher kann die Gesamtuntersuchung als erstmaliger Versuch gewertet werden, den Umgang mit Leistung im Kontext von Kompetenzorientierung in der Berufsschule zu beleuchten. Auf Forschungsergebnisse aus anderen Schultypen, die bereits vorliegen, wird hier auf Grund der fehlenden Vergleichbarkeit nicht eingegangen.

\section{Design und Ablauf}


Die Kategorienbildung, die im ersten Teil der Untersuchung mittels sechs leitfadengestützten Interviews mit niederösterreichischen Berufsschullehrer*innen erhoben worden war, diente der Überprüfung und Verdichtung der anfänglichen Problemstellung sowie zur Erhebung ergänzender Fragen (vgl. Tabelle 1). Deren Auswertungsergebnisse mündeten ihrerseits in die Erarbeitung eines empirisch-quantitativen Fragebogens, welcher, nach Erteilung der Genehmigung durch die Bildungsdirektion NÖ, mit der Bitte um Beantwortung an alle niederösterreichischen Berufsschullehrer*innen versendet wurde. Diesbezüglich kann von einem Sequential Mixed Design gesprochen werden, da sich die quantitativ erhobenen Daten des Fragebogens auf die qualitativ gewonnen Ergebnisse der ersten Erhebung beziehen (Teddlie und Tashakkori, 2006, S. 153).

\begin{tabular}{|l|l|}
\hline \multicolumn{1}{|c|}{ Kategorien } & \multicolumn{1}{c|}{ Subkategorien } \\
\hline K1 Kompetenzorientierter Unterricht (KOMPU) & $\begin{array}{l}\text { SK1 Individuelle Deutung von KOMPU } \\
\text { SK2 Wissen um KOMPU }\end{array}$ \\
\hline K2 Leistungsfeststellung & $\begin{array}{l}\text { SK1 Wissen um kompetenzorientierte Leistungsfeststellung } \\
\text { SK2 Anwendung von kompetenzorientierten } \\
\text { Leistungsfeststellungsmethoden }\end{array}$ \\
\hline K3 Leistungsbeurteilung & $\begin{array}{l}\text { SK1 Wissen um kompetenzorientierte Leistungsbeurteilung } \\
\text { SK2 Anwendung von kompetenzorientierten } \\
\text { Leistungsbeurteilungsmethoden }\end{array}$ \\
\hline K4 Fortbildungswünsche & \\
\hline K5 Wissen um schulrechtliche Grundlagen & \\
\hline
\end{tabular}

Tabelle 1: Kategorien der qualitativen Analyse (Lehmann \& Erhard, 2020)

\section{Deskriptive Analyse}

Insgesamt wurden in diesem Fragebogen 41 Fragen im Aussageformat formuliert und auf einer vierteiligen Ratingskala (1...trifft völlig zu, 2...trifft eher zu, 3...trifft eher nicht zu, 4...trifft gar nicht zu) bewertet. Die Fragen selbst stützen sich auf die Ergebnisse der vorgeschalteten leitfadengestützten Experteninterviews, die sowohl aus der relevanten Literatur erhobene deduktive Themen enthielten als auch durch die im Zuge des Interviews induktiv ermittelten Items ergänzt wurden. Aus diesem Grund konnte auf einen Pretest verzichtet werden. Einleitend wurde zudem auf die Achtung der Anonymität aller teilnehmenden Lehrkräfte hingewiesen.

Für die Erstellung und Distribution des Fragebogens wurde das Online-Tool LimeSurvey gewählt, das in einem Zeitfenster von 14 Tagen für alle niederösterreichischen Berufsschullehrer*innen von Ende Mai bis Anfang Juni 2020 zur Beantwortung geöffnet war.

Die Stichprobe zur Erhebung des quantitativen Datenmaterials mittels Fragebogen umfasste die Gruppe aller niederösterreichischen Berufsschullehrer*innen, welche zum Zeitpunkt der Erhebung 554 Lehrkräfte zählte, wobei das Berufsschulwesen in Niederösterreich zurzeit 16 Landesberufsschulen und zwei Exposituren umfasst. Nach Beendigung der Befragung konnten 139 Fragebögen gezählt werden, wobei 107 Fragebögen vollständig und 32 teilweise ausgefüllt worden waren. In seiner Gesamtheit belief sich der Rücklauf daher auf 25,1\%.

Die statistische Bearbeitung und Analyse der erhobenen Daten erfolgte mit dem Statistikprogramm SPSS, Version 26 (vgl. IBM Corporation 2020). Die Verteilung der Häufigkeiten der Antworten wurde für die jeweiligen Fragen in Histogrammen dargestellt. Die Korrelation zwischen den Fragepaaren wurde mittels Korrelationskoeffizienten nach Pearson ( $r$ ) überprüft. Die jeweiligen Signifikanzniveaus sind im jeweiligen Unterkapitel dargestellt.

Folgende Fragen wurden in Korrelation gesetzt:

\begin{tabular}{|l|l|l|}
\hline Kapitel & Frage & Frage \\
\hline
\end{tabular}


R\&E-SOURCE https://journal.ph-noe.ac.at

\begin{tabular}{|c|c|c|c|c|}
\hline 5.1 .1 & 10 & $\begin{array}{l}\text { Mir sind unterschiedliche Erarbeitungs- } \\
\text { formate bekannt, wie z. B. Projekt- } \\
\text { arbeiten, Präsentationen oder Portfolio }\end{array}$ & 11 & $\begin{array}{l}\text { Wenn ja: Wenden Sie diese } \\
\text { überwiegend (> } 50 \% \text { ) an? }\end{array}$ \\
\hline Kapitel & Frage & & Frage & \\
\hline 5.1 .2 & 12 & $\begin{array}{l}\text { Mir sind unterschiedliche kompetenz- } \\
\text { orientierte } \\
\text { Leistungsfeststellungsformate bekannt, } \\
\text { wie z. B. Multiple Choice bei } \\
\text { Formulierungsschwierigkeiten oder } \\
\text { ausschließlich mündliche Prüfungs- } \\
\text { formate für SuS mit Lese- und Recht- } \\
\text { schreibschwäche. }\end{array}$ & 13 & $\begin{array}{l}\text { Wenn ja: Wenden Sie diese } \\
\text { o. ä. Formate individuell an? }\end{array}$ \\
\hline 5.1 .3 & 14 & $\begin{array}{l}\text { Mir ist bekannt, dass ein Kompetenz- } \\
\text { raster als Leistungsfeststellungs- } \\
\text { instrument der Mitarbeit eingesetzt } \\
\text { werden kann. }\end{array}$ & 15 & $\begin{array}{l}\text { Wenn ja: Verwenden Sie einen } \\
\text { Kompetenzraster als } \\
\text { Leistungsfeststellungsformat? }\end{array}$ \\
\hline 5.1 .4 & 16 & $\begin{array}{l}\text { Mir ist bekannt, dass die Mitarbeitsnote } \\
\text { in einem Gegenstand ohne Schularbeit } \\
\text { die alleinige Grundlage der Jahres- } \\
\text { beurteilung sein kann. }\end{array}$ & 17 & $\begin{array}{l}\text { Wenn ja: Nützen Sie dieses } \\
\text { Prinzip der } \\
\text { Leistungsbeurteilung? }\end{array}$ \\
\hline 5.1 .5 & 18 & $\begin{array}{l}\text { Mir ist die Überführung der Ergebnisse } \\
\text { eines Kompetenzrasters in die fünf } \\
\text { Notenschritte bekannt. }\end{array}$ & 19 & $\begin{array}{l}\text { Wenn ja: Nützen Sie einen } \\
\text { Kompetenzraster als Grund- } \\
\text { lage für die Feststellung der } \\
\text { fünf Notenschritte? }\end{array}$ \\
\hline 5.1 .6 & 20 & $\begin{array}{l}\text { Mir ist bekannt, dass in einem } \\
\text { Theoriegegenstand ohne Schularbeit } \\
\text { ausschließlich die Ergebnisse von } \\
\text { Kompetenzrastern als Instrument zur } \\
\text { Leistungsbeurteilung (Notengebung) am } \\
\text { Ende des Lehrganges dienen können. }\end{array}$ & 21 & $\begin{array}{l}\text { Wenn ja: Wenden Sie diese Art } \\
\text { der Leistungsbeurteilung an? }\end{array}$ \\
\hline
\end{tabular}

Tabelle 2: Korrelationspaare

Darüber hinaus wurde eine weitere Gruppe erstellt, die Fragen und Antworten zum Grundlagenverständnis im Bereich Kompetenzorientierung zusammenfassen sollte. Die Ergebnisse der Fragen (vgl. Tab. 3) zeigen mögliche Ursachen in Bezug auf das abgebildete Verhältnis zwischen dem Wissen um kompetenzorientierte Leistungsfeststellung und Leistungsbeurteilung und dessen Umsetzung.

\begin{tabular}{|l|ll|}
\hline Kapitel & Frage & \\
\hline 5.2 .1 & 28 & Ich wurde bei der Umsetzung kompetenzorientierter Lehrpläne miteinbezogen. \\
\hline 5.2 .2 & 29 & \begin{tabular}{l} 
Ich wurde über die Kerninhalte kompetenzorientierter Leistungsfeststellung informiert \\
\hline 5.2 .3
\end{tabular} \\
\hline 5.2 .4 & 31 & $\begin{array}{l}\text { Ich wurde über die Kerninhalte kompetenzorientierter Leistungsbeurteilung informiert. } \\
\text { Leistungsfeststellung (Prüfungsformate). }\end{array}$ \\
\hline 5.2 .5 & 32 & $\begin{array}{l}\text { Ich informiere mich laufend selbst über kompetenzorientierte Methoden der } \\
\text { Leistungsbeurteilung (wie z. B. Kompetenzraster). }\end{array}$ \\
\hline 5.2 .6 & 38 & $\begin{array}{l}\text { Mir wird an der Schule bei Fragen zu kompetenzorientierter Leistungsfeststellung } \\
\text { geholfen. }\end{array}$ \\
\hline 5.2 .7 & 39 & $\begin{array}{l}\text { Mir wird an der Schule bei Fragen zu kompetenzorientierter Leistungsbeurteilung } \\
\text { geholfen. }\end{array}$ \\
\hline
\end{tabular}

Tabelle 3: Fragen zum Grundlagenverständnis 


\subsection{Zusammenhang zwischen Wissen und Anwendung im Bereich Leistung im Kontext von Kompetenzorientierung}

In diesem Abschnitt wurde das Wissen um und die Anwendung von Leistung im Kontext von Kompetenzorientierung im Rahmen einer deskriptiven Analyse verglichen.

Dabei wurden diese beiden Bereiche anhand des jeweiligen der Frage zugeordneten Histogramms in Prozent beschrieben, um damit mögliche Zusammenhänge zwischen Wissen und Anwendung sichtbar zu machen.

Im Folgenden wurden für jedes Fragepaar der Korrelationskoeffizient nach Pearson ( $r$ ) und die Signifikanz ( $p$ ) dargestellt.

\subsubsection{Zusammenhang zwischen Wissen und Anwendung in Bezug auf offene Erarbeitungsformate, wie z. B. Projektarbeit, Präsentation oder Portfolio}

In diesem Bereich konnte ein schwacher statistischer Zusammenhang erkannt werden $(r=0,082 ; p>0,05)$. Darüber hinaus gaben $97 \%$ der Lehrkräfte an, völlig oder eher Kenntnisse über die unterschiedlichen offenen Erarbeitungsformate zu haben (vgl. Abb.1). 26 \% der Befragten führten in Bezug auf die Frage „Wenn ja: Wenden Sie diese überwiegend (> 50\%) an?" an, dieses Wissens eher nicht bzw. gar nicht anzuwenden (vgl. Abb.2).

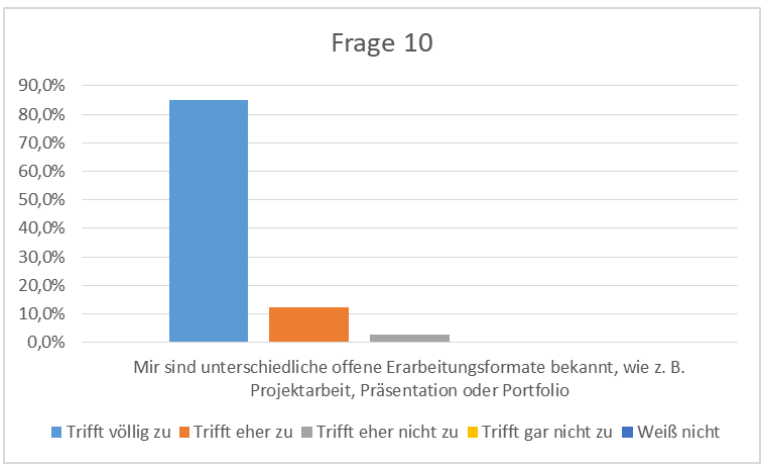

Abbildung 1: Frage 10

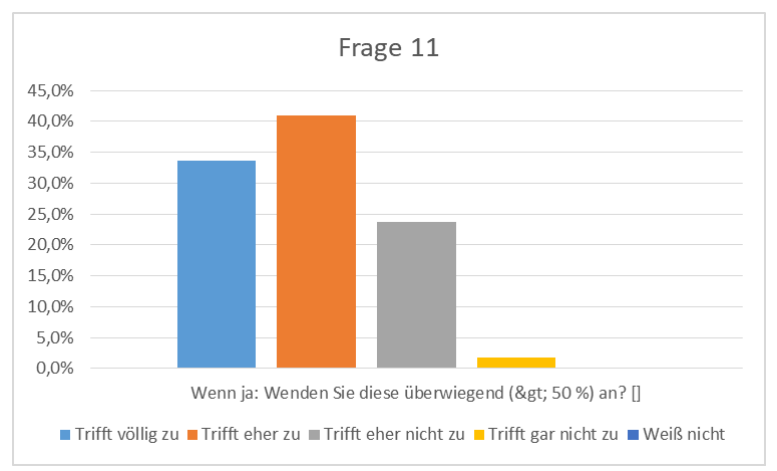

Abbildung 2: Frage 11

\subsubsection{Zusammenhang zwischen Wissen und Anwendung in Bezug auf kompetenzorientierte Leistungsfeststellungsformate, wie z. B. Multiple-Choice-Tests}

In diesem Bereich zeigte sich ein gemäßigter statistischer Zusammenhang nach Pearson $(r=0,337 ; p>0,001)$. $83 \%$ der Lehrkräfte führten an, völlig oder eher Kenntnisse über unterschiedliche kompetenzorientierte Leistungsfeststellungsformate zu haben (vgl. Abb.3). Zu der Frage der Anwendung dieser Formate gaben $23 \%$ aller Befragten an, diese Formate eher nicht bzw. gar nicht anzuwenden (vgl. Abb.4). 


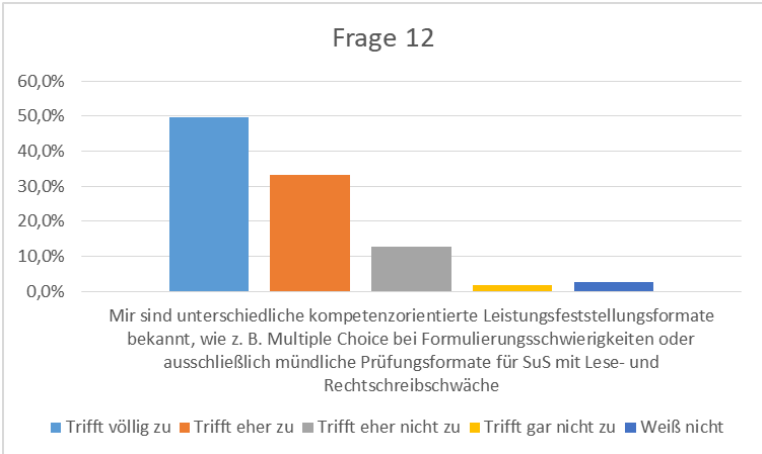

Abbildung 3: Frage 12

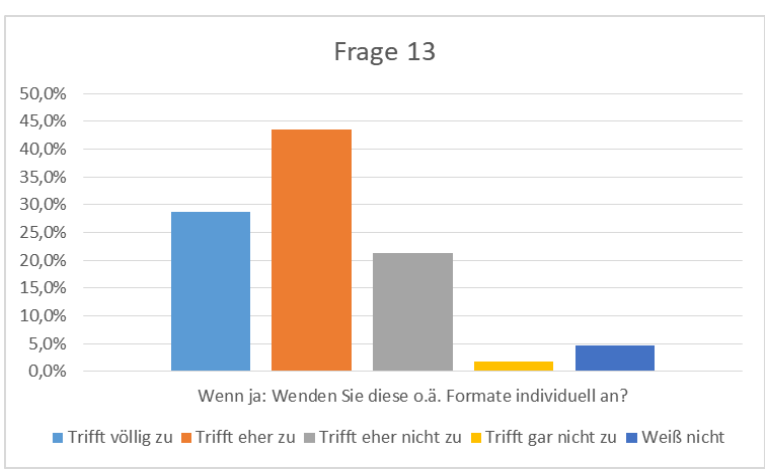

Abbildung 4: Frage 13

\subsubsection{Zusammenhang zwischen Wissen und Anwendung in Bezug auf kompetenzorientierte Leistungsfeststellungsformate (z. B. Kompetenzraster)}

In diesem Bereich zeigte sich ebenfalls ein gemäßigter statistischer Zusammenhang nach Pearson $(r=0,424 ; p>$ 0,00002). Hierbei ließ sich erkennen, dass $79 \%$ der Lehrkräfte ihr Wissen in Bezug auf den Kompetenzraster als kompetenzorientiertes Leistungsfeststellungsinstrument der Mitarbeit (vgl. Abb.5) als vollständig bzw. eher vollständig angaben, wohingegen $48 \%$ der Teilnehmer*innen vermerkten, einen Kompetenzraster als Instrument der Leistungsfeststellung eher nicht bzw. gar nicht einzusetzen (vgl. Abb.6).

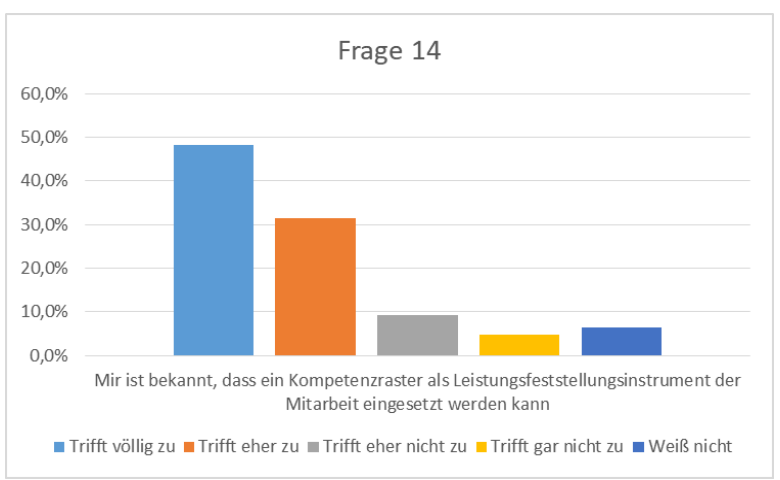

Abbildung 5: Frage 14

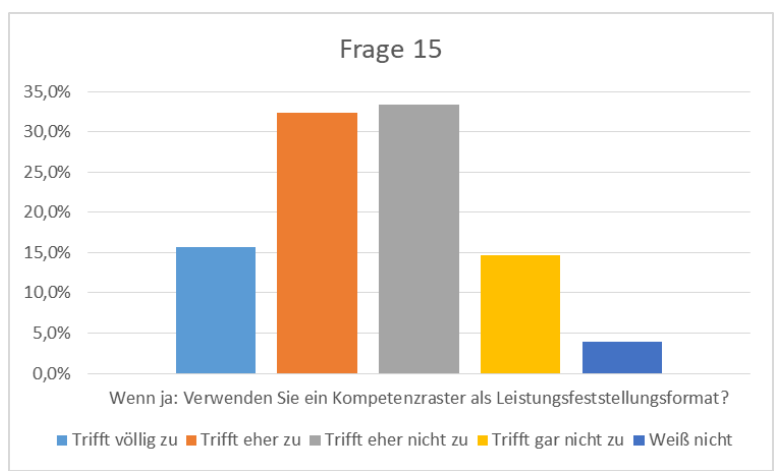

Abbildung 6: Frage 15

\subsubsection{Zusammenhang zwischen dem Wissen um die Mitarbeitsnote als wichtigstes kompetenzorientiertes Leistungsbeurteilungsformat und deren tatsächliche Anwendung}

Auch hier ließ sich ein gemäßigter statistischer Zusammenhang erkennen ( $r=0,312 ; p>0,001)$. Was das Wissen um die Mitarbeitsnote als das herausragende kompetenzorientierte Leistungsbeurteilungsformat in Gegenständen ohne Schularbeit betrifft, gaben 88 \% der Lehrkräfte an, völlig oder eher völlig darüber Kenntnis zu haben (vgl. Abb.7). $37 \%$ der Befragten führten an, die Mitarbeitsnote als alleiniges Leistungsbeurteilungsinstrument in Gegenständen ohne Schularbeiten eher nicht oder gar nicht einzusetzen (vgl. Abb.8). 


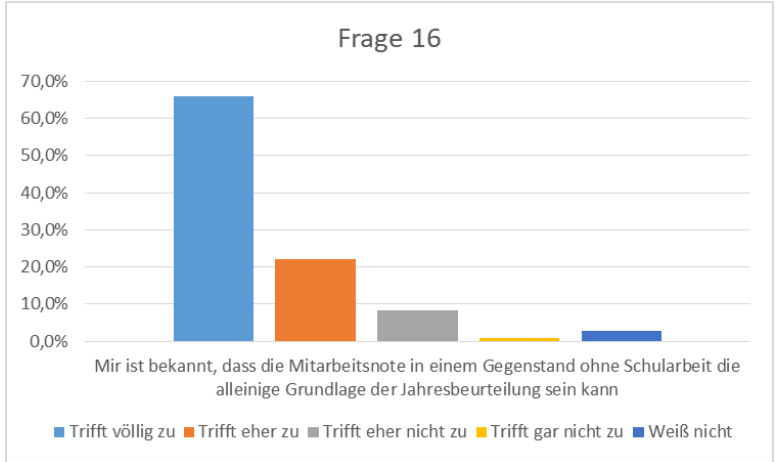

Abbildung 7: Frage 16

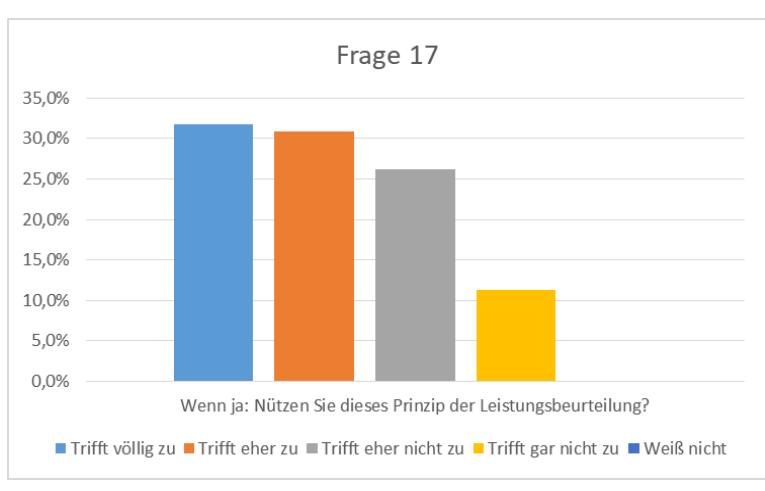

Abbildung 8: Frage 17

\subsubsection{Zusammenhang zwischen dem Wissen und der Anwendung in Bezug auf die Überführung der Ergebnisse eines Kompetenzrasters in die 5-teilige Notenskala}

In der Korrelation von Frage 18 und 19 zeigte sich ein starker statistischer Zusammenhang $(r=0,753 ; p>0,000)$. $64 \%$ der Lehrkräfte gaben an, völlig oder eher völlig Kenntnis über die Überführung der Ergebnisse eines Kompetenzrasters in die 5-teilige Notenskala zu haben (vgl. Abb.9). Bei 53 \% der Beteiligten wurde auf die Frage der Anwendung dieses Wissens mit eher nicht oder gar nicht geantwortet (vgl. Abb.10).

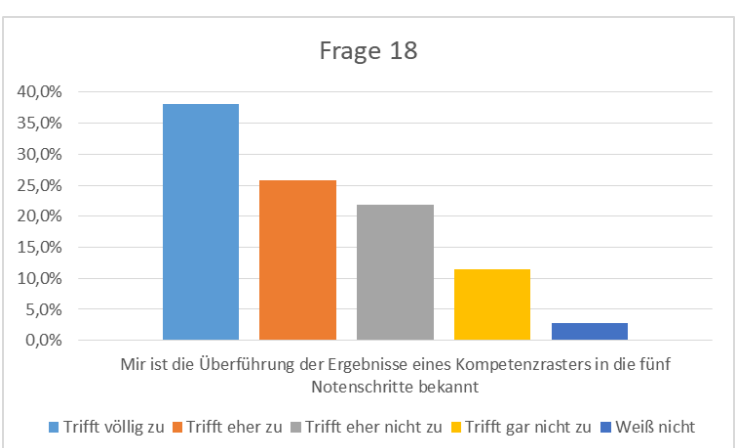

Abbildung 9: Frage 18

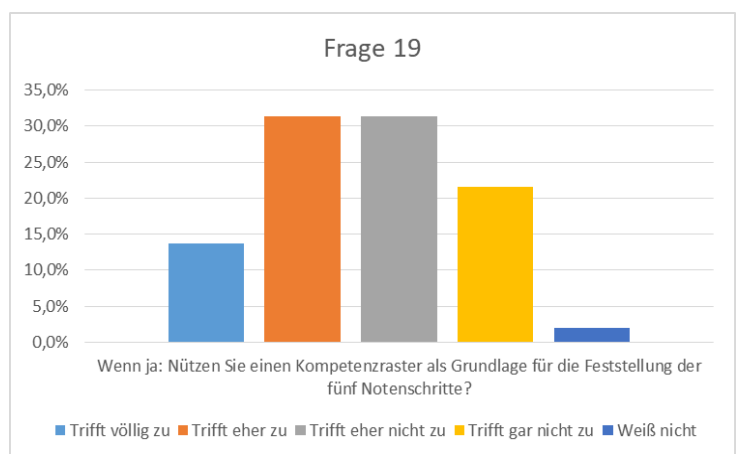

Abbildung 10: Frage 19

\subsubsection{Zusammenhang zwischen dem Wissen um und der Anwendung von Kompetenzraster als Instrument der Notengebung}

Zwischen Frage 20 und 21 zeigte sich wiederum ein gemäßigter statistischer Zusammenhang $(r=0,562 ; p>$ 0,001). Dabei führten 66 \% der Lehrkräfte an, völlig oder eher völlig über die Anwendung von Kompetenzrastern als Instrument der Notengebung in Nicht-Schularbeitsgegenständen Bescheid zu wissen (vgl. Abb.11). 55 \% der Teilnehmer*innen gaben zu erkennen, dieses Instrument der kompetenzorientierten Leistungsbeurteilung eher nicht oder gar nicht anzuwenden (vgl. Abb.12). 


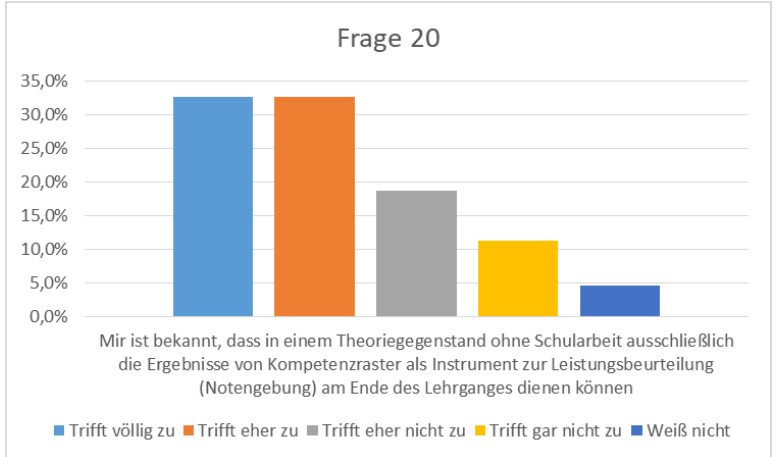

Abbildung 11: Frage 20

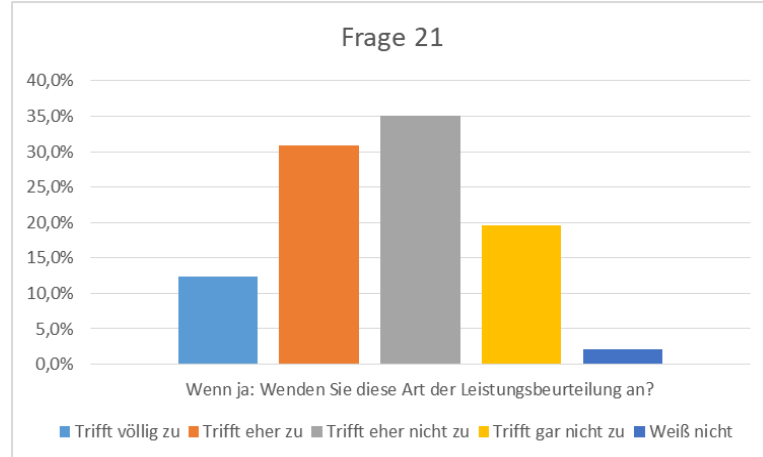

Abbildung 12: Frage 21

Die unterschiedlich starken Zusammenhänge zwischen Wissen und Anwendung sind im Kapitel 6 mit den Ergebnissen der qualitativen Analyse in Beziehung gesetzt.

\subsection{Grundlagenverständnis Kompetenzorientierung}

\subsubsection{Einbeziehung der Lehrkraft in die Erarbeitung kompetenzorientierter Lehrinhalte}

$72 \%$ der teilnehmenden Lehrkräfte gaben an, bei der Umsetzung kompetenzorientierter Lehrpläne völlig oder eher völlig miteinbezogen worden zu sein, im Vergleich zu 16 \%, die ihrer Meinung nach eher nicht oder gar nicht eingebunden worden waren (vgl. Abb.15).

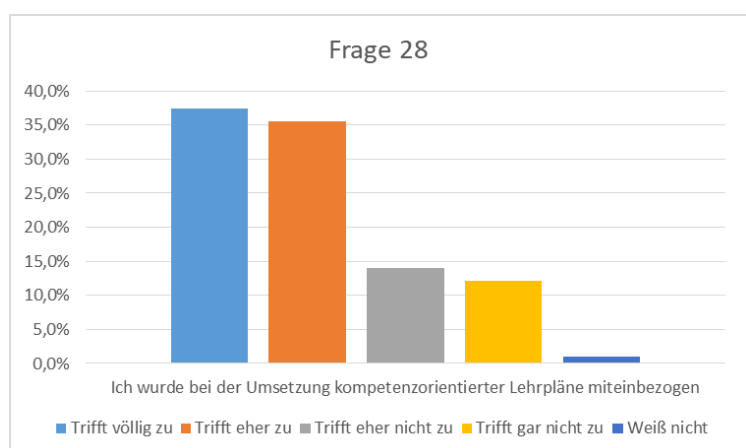

Abbildung 15: Frage 28

\subsubsection{Subjektiv erlebtes Maß an Informationen über kompetenzorientierte Kerninhalte zur Leistungsfeststellung und -beurteilung}

In Bezug auf die kompetenzorientierte Leistungsfeststellung führten $78 \%$ der Teilnehmer*innen an, über kompetenzorientierte Kerninhalte völlig oder eher völlig informiert worden zu sein, wohingegen $20 \%$ angaben, eher nicht oder gar nicht informiert worden zu sein (vgl. Abb.16).

In Bezug auf die kompetenzorientierte Leistungsbeurteilung gaben 77 \% der befragten Lehrkräfte an, völlig oder eher völlig über die Kerninhalte informiert worden zu sein. $12 \%$ der Teilnehmer*innen wollen diesbezüglich eher nicht oder gar nicht informiert worden sein (vgl. Abb.17). 


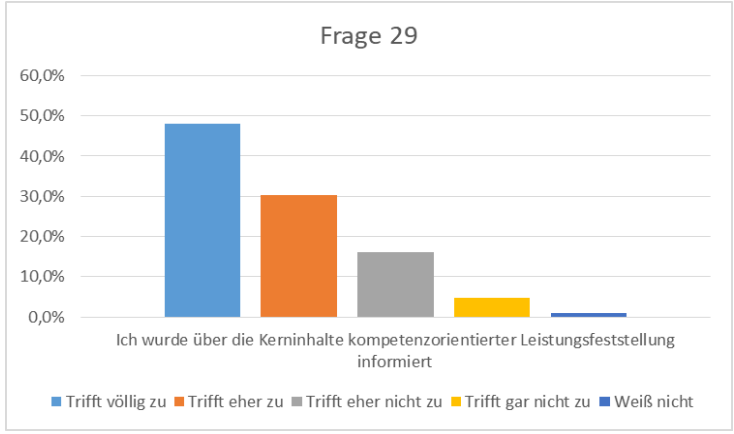

Abbildung 16: Frage 29

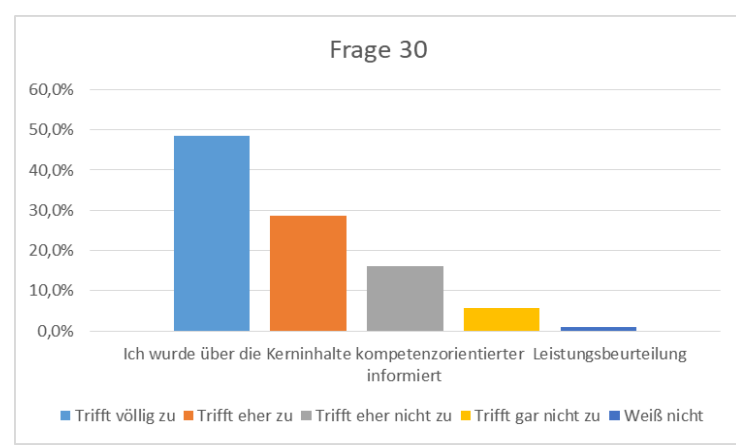

Abbildung 17: Frage 30

\subsubsection{Fortbildungsbestrebungen in Bezug auf kompetenzorientierte Leistungs- feststellungs- und Leistungsbeurteilungsmethoden}

Bezüglich kompetenzorientierer Leistungsfeststellungsmethoden führten $64 \%$ der Teilnehmer*innen an, sich völlig oder eher völlig über kompetenzorientierte Kerninhalte informiert zu haben, wohingegen $33 \%$ angaben, sich diesbezüglich eher nicht oder gar nicht Informationen einzuholen (vgl. Abb.18).

In Bezug auf kompetenzorientierte Leistungsbeurteilungsmethoden zeigte sich, dass $57 \%$ der Teilnehmer*innen sich völlig oder eher völlig über kompetenzorientierte Kerninhalte informieren. $43 \%$ der Lehrkräfte gaben an, sich diesbezüglich eher nicht oder gar nicht zu informieren (vgl. Abb.19).

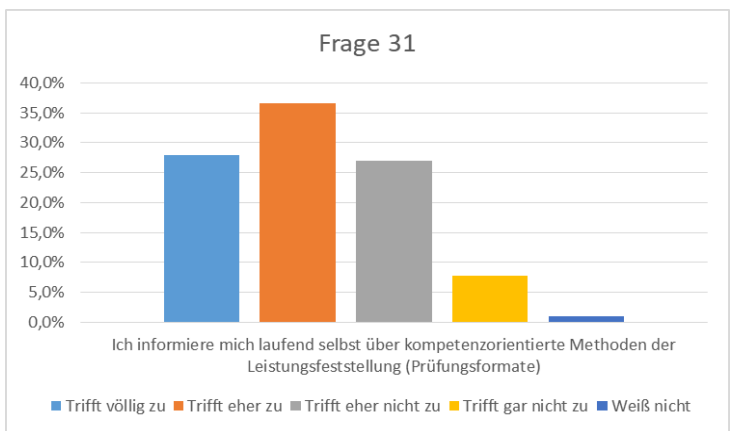

Abbildung 18: Frage 31

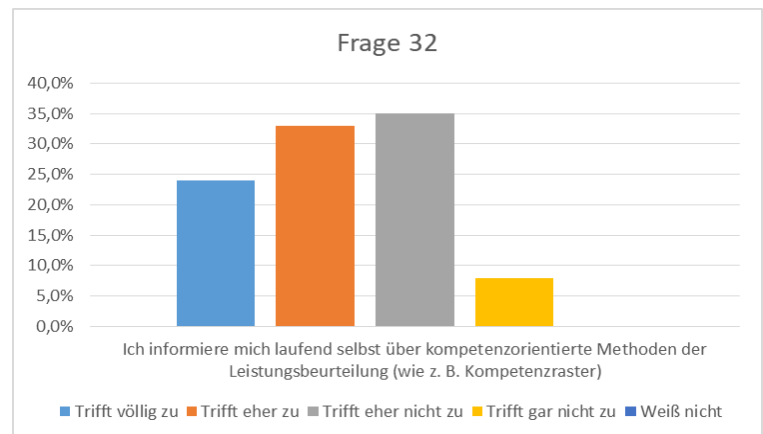

Abbildung 19: Frage 32

\subsubsection{Erlebte Hilfestellungen im Bereich kompetenzorientierter Leistungsfeststellungs- und Leistungsbeurteilungsmethoden}

$73 \%$ der teilnehmenden Lehrkräfte fühlten sich in Bezug auf Fragen zu kompetenzorientierter Leistungsfeststellung völlig oder eher völlig unterstützt, im Vergleich zu $25 \%$, denen ihrer Meinung nach eher nicht oder gar nicht geholfen wurde (vgl. Abb.20).

In Bezug auf Fragen zu kompetenzorientierter Leistungsbeurteilung gaben 73 \% der Befragten an, völlig oder eher völlig unterstützt zu werden. 23 \% dagegen empfanden das eher nicht oder gar nicht so (vgl. Abb.21). 


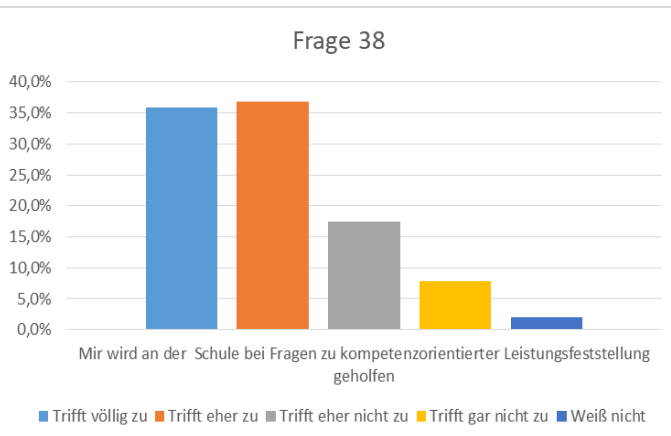

Abbildung 20: Frage 38

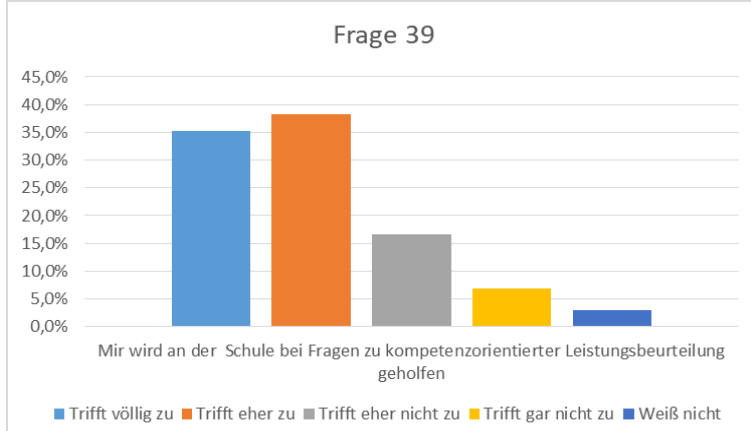

Abbildung 21: Frage 39

Die Ergebnisse zum Grundlagenverständnis bezüglich Kompetenzorientierung zeigen, dass ca. drei Viertel der Teilnehmenden sich informiert fühlen bzw. auch entsprechende Hilfestellungen erlebt haben. Die Bestrebungen, sich laufend fortzubilden, bleiben hinter dieser Rate allerdings zurück.

\section{Zusammenführung der Ergebnisse}

Im folgenden Abschnitt wird ein Überblick über die Zusammenführung der quantitativen und qualitativen Ergebnisse gegeben. Die daraus resultierenden Erkenntnisse wurden abschließend in Bezug auf mögliche noch nicht genannte Einflüsse diskutiert, um einen entsprechenden Ausblick ableiten zu können.

\subsection{Wissen - Grundlagen Kompetenzorientierung}

$7 \%$ der teilnehmenden Lehrkräfte gaben an, bei der Umsetzung kompetenzorientierter Grundlagen in Form von Lehrplänen völlig oder eher völlig miteinbezogen worden zu sein, im Vergleich zu $16 \%$, die ihrer Meinung nach eher nicht oder gar nicht eingebunden worden waren (vgl. Abb.15).

Was das Wissen um kompetenzorientierte Kerninhalte der Leistungsfeststellung betraf, führten $78 \%$ der Teilnehmer*innen an, völlig oder eher völlig informiert worden zu sein, wohingegen 20 \% der Befragten angaben, eher nicht oder gar nicht informiert worden zu sein (vgl. Abb.16).

Hinsichtlich des Wissens um Kerninhalte kompetenzorientierter Leistungsbeurteilung gaben $77 \%$ der befragten Lehrkräfte an, völlig oder eher völlig über diese informiert worden zu sein. 12 \% der Teilnehmer*innen wollen diesbezüglich eher nicht oder gar nicht informiert worden sein.

Bezüglich der Grundlagenvermittlung kompetenzorientierter Unterrichtsprinzipien konnten innerhalb der Gruppe der in Einzelinterviews befragten Lehrkräfte zwei Pole ausgemacht werden. Drei Lehrkräfte empfanden die formale Einschulung in kompetenzorientierter Unterrichtsorganisation, Leistungsfeststellung und Leistungsbeurteilung als nicht vorhanden bis unzureichend. Drei der befragten Lehrkräfte gaben an, entweder über die Grundausbildung bzw. Seminare bzw. schulinternen Austausch ausreichend zu ihrem Wissen über kompetenzorientierte Unterrichtsprinzipien gekommen zu sein.

\subsection{Wissen und Anwendung - Kompetenzorientierte Erarbeitungsformate}

In Bezug auf ihr Wissen um offene Erarbeitungsformate gaben $85 \%$ der Teilnehmer*innen an, dass ihnen diese Formate völlig bekannt sind. Eine hohe Zustimmung zeigte sich auch in der qualitativen Erhebung, wo sechs von sechs der Befragten diese Art der kompetenzorientierten Erarbeitung von Unterrichtsinhalten kannte (vgl. Abb.1). 
Die Anwendung dieses Wissens bejahten $34 \%$ der Teilnehmer*innen völlig und $41 \%$ eher. In der qualitativen Auswertung gaben sechs von sechs Befragten an, Methoden einer offenen Erarbeitung selbst zu verwenden, was ebenfalls einer hohen Übereinstimmung der Aussagen entsprach (vgl. Abb. 2).

\subsection{Wissen und Anwendung - Kompetenzorientierte Leistungsfeststellungs-formate}

Kompetenzorientierte Leistungsfeststellungsformate für heterogene Schülergruppen wurden von $50 \%$ der Lehrkräfte als völlig bekannt und von $33 \%$ als eher bekannt ausgewiesen (vgl. Abb. 3), wobei $29 \%$ der Teilnehmer*innen angaben, diese Formate völlig anzuwenden, und $44 \%$ anführten, eher differenzierende Maßnahmen zu ergreifen (vgl. Abb. 4).

In den Ergebnissen der Einzelinterviews gaben vier von sechs Befragten an, als Maßnahme bei der Feststellung von Leistung in heterogenen Klassen das Leistungsniveau an die Sozialnorm anzupassen und dementsprechend abzusenken.

Als Beispiel für ein kompetenzorientiertes Leistungsfeststellungsformat führten $48 \%$ der befragten Lehrer*innen an, einen Kompetenzraster völlig zu kennen, und $31 \%$ von ihnen, diesen eher zu kennen (vgl. Abb.5). $16 \%$ der Fragebogenteilnehmer*innen erklärten, einen Kompetenzraster zu verwenden (stimme völlig zu), $32 \%$ diesen eher und $33 \%$ eher nicht zu verwenden (vgl. Abb.6).

In der qualitativen Untersuchung stimmten hingegen sechs der sechs Befragten zu, dieses Instrument zu kennen, wobei nur ein*e Interviewteilnehmer*in bestätigte, einen Kompetenzraster tatsächlich als Leistungsfeststellungsinstrument zu verwenden.

\subsection{Wissen und Anwendung - Kompetenzorientierte Leistungsbeurteilung}

Die deskriptive Darstellung der Fragebogenerhebung zeigte, dass $66 \%$ der Lehrkräfte der Meinung waren, dass die Mitarbeitsnote in den Gegenständen ohne Schularbeiten als alleinige Grundlage zur Notenfindung völlig ausreichen würde, $22 \%$ stimmten dem eher zu (vgl. Abb.7).

Was die Anwendung betraf, gaben nur $32 \%$ der Fragebogenteilnehmer*innen an, diese kompetenzorientierte Maßnahme im Einklang mit der LBVO (1974, i.d.g.F.) vollständig zu ergreifen, 31 \% eher und 26 \% eher nicht (vgl. Abb.8). Dem entsprachen die Ergebnisse der Einzelinterviews, bei denen zwei der sechs Befragten anführten, dass sie alle Leistungsfeststellungen in schularbeitsfreien Gegenständen in der Mitarbeitsnote zum Ausdruck bringen würden.

Was das spezielle Wissen um den Kompetenzraster als Beurteilungsinstrument im Zusammenspiel mit dem Notenschlüssel betraf, wiesen $38 \%$ der Teilnehmer*innen aus, völlige Kenntnisse darüber zu haben, $26 \%$ eher und $22 \%$ eher nicht (vgl. Abb.9), wohingegen alle sechs Befragten der Einzelinterviews zustimmten, diesen Anwendungsbereich des Kompetenzrasters zu kennen.

In Bezug auf die Anwendung zeigten sich jedoch ähnliche Ergebnisse, wenn $14 \%$ der Fragebogenteilnehmer*innen völlig zustimmten, dieses Leistungsbeurteilungsinstrument anzuwenden, $31 \%$ eher, $31 \%$ eher nicht und $22 \%$ gar nicht (vgl. Abb. 10), da nur eine interviewte Lehrkraft erklärte, den Kompetenzraster parallel zur Notenstufenskala zu verwenden.

Darüber hinaus führten 66 \% der Lehrkräfte bei der Fragebogenerhebung an, völlig oder eher völlig über die Anwendung von Kompetenzrastern als Instrument der Notengebung in Nicht-Schularbeitsgegenständen, Bescheid zu wissen (vgl. Abb.20). $55 \%$ der Teilnehmer*innen gaben an, dieses Instrument der kompetenzorientierten Leistungsbeurteilung eher nicht oder gar nicht anzuwenden (vgl. Abb.21).

Fünf von sechs in den Einzelinterviews befragten Lehrkräften sahen im Kompetenzraster noch kein geeignetes Leistungsbeurteilungsinstrument im Rahmen von Nicht-Schularbeitsgegenständen. 


\subsection{Fortbildungswünsche}

Fünf von sechs der Befragten wünschten sich im Rahmen der qualitativen Erhebung entsprechende Weiterbildung in diesem Bereich, wobei auch der kollegiale Austausch schulintern, aber auch schulübergreifend als wichtiger Ideenpool genannt wurde. In einem Interview zeigte sich eine Lehrkraft mit dem derzeitigen Wissensstand in diesem Bereich zufrieden. Auch in der Fragebogenerhebung wurde mehrheitlich der Wunsch (völlig und eher) nach mehr Information zu Methoden der kompetenzorientierten Leistungsfeststellung (64,4 \%; vgl. Abb.18) und Leistungsbeurteilung (57\%; vgl. Abb. 19) geäußert.

\section{Diskussion und Ausblick}

Insgesamt konnte beobachtet werden, dass das Wissen um kompetenzorientierte Methoden vor allem dort sehr hoch zu sein scheint, wo Lehrkräfte dieses Wissen auch zur Anwendung bringen müssen, so zum Beispiel in der Umsetzung von kompetenzorientierten Lehrplänen, Unterrichtsvorbereitungen und Unterrichtsmaterialien, an denen sie selbst mitgearbeitet haben.

Darüber hinaus liegt die Vermutung nahe, dass die Methodenvielfalt in Bezug auf die Entwicklung von Leistungsfeststellungsformaten steigt, je mehr organisatorischen und methodischen Spielraum die Lehrkraft hat und je mehr die berufsspezifischen Eigenheiten in die Unterrichtsgestaltung einfließen dürfen.

Auffallend war das Verständnis der Befragten in Bezug auf die Gewichtung der Mitarbeitsbeurteilung als wichtige kompetenzorientierte Leistungsbeurteilungsmaßnahme im Verhältnis zu anderen Beurteilungsformen. Der überwiegende Teil aller Befragten gab nämlich an, zu wissen, dass die Mitarbeitsnote in den Gegenständen ohne Schularbeiten als alleinige Grundlage zur Notenfindung ausreicht. Trotzdem wurde dieses Beurteilungsformat nicht durchgängig von den Lehrkräften angewendet, obwohl die aktuelle LBVO gerade die Mitarbeit als wesentliche Säule der kompetenzorientierten Leistungsfeststellung ausweist.

Hier kann möglicherweise eine Orientierung an den Vorgaben der Direktion bzw. der Bildungsdirektion angenommen werden, die tatsächlich oder im Erleben der betreffenden Lehrkräfte die eigene Autonomie bezüglich der Leistungsbeurteilung einschränkt.

Die Notwendigkeit vor allem in den Lehrgangsberufsschulen (10 Wochen oder kürzer) zu einer raschen, rechtlich fundierten Notenfindung kommen zu müssen, zeigte sich in der Datenlage als starke Ausrichtung auf den traditionellen Rahmen bildungsorientierter Leistungsfeststellungsstandards. Hier scheinen die Lehrkräfte neben den vorgeschriebenen Schularbeiten mehrheitlich Testnoten (besondere schriftliche Überprüfungen; Anm. der Autorin*des Autors) gleichermaßen oder mit höherem Anteil gegenüber der Mitarbeit als Benotungsgrundlage heranzuziehen.

Der Kompetenzraster als wesentliche Säule der kompetenzorientierten Leistungsfeststellung und Grundlage der Leistungsbeurteilung scheint mehrheitlich noch kein geeignetes Instrument in Nicht-Schularbeitsgegenständen darzustellen.

In diesem Zusammenhang war aus den Ergebnissen zu erkennen, dass Prüfungs- und Benotungsgebote, die an dem rein bildungsorientierten Unterricht der Vergangenheit ausgerichtet sind, nach wie vor einen maßgeblichen Einfluss auf die Methodenwahl und damit auf die eher geringe Anwendung kompetenzorientierter Unterrichtsmethoden haben.

Zudem kann das erkennbare Missverhältnis zwischen Wissen und Anwendung kompetenzorientierter Leistungsfeststellung und Leistungsbeurteilung in der unterschiedlichen Verfügbarkeit bzw. Inanspruchnahme einschlägiger Aus- und Weiterbildungsangebote geortet werden. Hier zeichnete sich in der qualitativen Erhebung ein Zusammenhang zwischen Dienstalter und Ausbildungsinhalten zur Kompetenzorientierung ab, wenn dienstjüngere Kolleg*innen auf die Grundausbildung als Fundament ihrer Kenntnisse über Kompetenzorientierung verweisen. Demgegenüber gab eine überwiegende Anzahl von Lehrkräften an, dass sie bei Bedarf auf Informationen von Kolleg*innen an der eigenen Schule oder auch an anderen Schulen zurückgreifen und sich dadurch unterstützt fühlen. Hier kann eine Kompensation von fehlender formaler Ausund Weiterbildung durch informelle Wissensübermittlung angenommen werden. 
Abschließend ist auf die Limitationen der vorliegenden Untersuchung hinzuweisen. Trotz der Tatsache, dass die qualitative Datenerhebung durch die Möglichkeit zur Rückfrage zu einer Vertiefung der Erkenntnislage beitragen konnte, muss eingeräumt werden, dass die Aussagekraft der quantitativen Auswertung eingeschränkt ist. Dies kann auf die Natur der hier verwendeten Querschnittsanalyse zurückgeführt werden, bei der Ergebnisse nicht kausal interpretierbar sind. Zudem konnte im Rahmen der Fragebogenerhebung nicht nach Dienstalter, Geschlecht, Berufsschultyp und Fachgruppe stratifiziert werden.

Wie wiederholt von den Teilnehmenden der leitfadengestützten Einzelinterviews betont wurde, hat die Berufsschule im Hinblick auf ihre naturgemäße praxisorientierte Ausrichtung die Anwendungstauglichkeit theoretischer Inhalte schon immer im Auge gehabt. Damit eine volle Entfaltung aller Aspekte dieses kompetenzorientierten Leitprinzips gelingen kann, gilt es, darauf zu achten, die Leistungsfeststellung und die Leistungsbeurteilung den didaktischen und methodischen Notwendigkeiten unterzuordnen und nicht umgekehrt. In diesem Sinne ist es von Bedeutung, die diversen Eigenheiten, die Berufsschulen aufgrund ihrer unterschiedlichen Zweige und Berufe aufweisen, zu identifizieren und diese Ressourcen insofern anzuerkennen, als die berufsspezifische Expertise der Lehrkräfte vermehrt einfließen darf. Dazu gehört auch, eine weitgehend autonome Anpassung der kompetenzorientierten Leistungsfeststellungs- und Leistungsbeurteilungsinstrumente im Rahmen der LBVO (1974, i.d.g.F.) an die spezifischen unterrichts- bzw. schulorganisatorischen Gegebenheiten zu ermöglichen.

Zum Erreichen dieses Zieles wären vor allem laufende Aus- und Weiterbildungsmaßnahmen anzudenken, die dem Lehrpersonal in Bezug auf eine kompetenzorientierte Lesart der LBVO Rechtsicherheit geben und gleichzeitig Spielraum zur Förderung des Einsatzes kompetenzorientierter Leistungsfeststellungs- und Leistungsbeurteilungsinstrumente (Kompetenzraster, Mitarbeitsprimat) einräumen.

\section{Literatur}

BMUKK (2012). Kompetenzorientiertes Unterrichten an berufsbildenden Schulen. Grundlagenpapier. Wien: Eigendruck BMUKK.

Dornmayer, H. \& Nowak, S. (2019). Lehrlingsausbildung im Überblick 2019. Strukturdaten, Trends und Perspektiven. Ibw-Forschungsbericht Nr.200. Wien: Eigendruck Institut für Bildungsforschung der Wirtschaft.

Europäische Union. (2009). Abgerufen am 28.09.2020 von https://www.cedefop.europa.eu/en/eventsandprojects/projects/european-credit-system-vocational-education-and-training-ecvet

Lehmann, E. \& Erhard, F. (2020). Über den Umgang mit schulischer Leistung unter kompetenzorientierten Gesichtspunkten. Eine Erhebung an niederösterreichischen Berufsschulen. R\&E-Source, Ausgabe S21, Baden.

Leistungsbeurteilungsverordnung (LBVO) vom 25.06. 1974 BGBL. 371/1974. Abgerufen am 20.06.2020 von https://www.ris.bka.gv.at/ Geltende Fassung.

Mohr, M. (2021). Abgerufen am 22.09.2021 von https://de.statista.com/statistik/daten/studie/882771/umfrage/berufsschulen-in-oesterreich/

Neuweg, G., H. (2019). Kompetenzorientierte Leistungsbeurteilung. Pädagogische und rechtliche Hilfestellungen für die Schulpraxis. Linz: TRAUNER Verlag.

Smigierski, J. (2018). Pearson-Korrelationskoeffizient in SPSS berechnen (Pearson's $r$ in SPSS). Abgerufen am 6.01.2021 von https://www.beratung-statistik.de

Teddlie, C. \& Tashakorri, A. (2020). Foundations of Mixed Methods Research: Integrating Quantitative and Qualitative Approaches in the Social and Behavioural Sciences. Thousand Oaks: SAGE Publications, Inc. 